\title{
Ambient Temperature Problem
}

National Cancer Institute

\section{Source}

National Cancer Institute. Ambient Temperature Problem. NCI Thesaurus. Code C63307.

Problem associated with compromised device performance at the ambient temperature or the storage at an inappropriate ambient temperature. 